\title{
COMPARISON OF MCUSUM AND GENERALIZED VARIANCE $|S|$ MULTIVARIATE CONTROL CHART PROCEDURE WITH INDUSTRIAL APPLICATION
}

\author{
M. S. HAMED ${ }^{1,2}$, A. A. EL SAYED ${ }^{1,3}$ and T. H. ABOUELMAGD ${ }^{1,2}$ \\ ${ }^{1}$ Department of Administrative Sciences \\ Faculty of Business Administration \\ Taibah University \\ Kingdom of Saudi Arabia \\ 2Department of Applied Statistics \\ Faculty of Commerce \\ Benha University \\ Egypt \\ e-mail: moswilem@gmail.com \\ ${ }^{3}$ Department of Statistics \\ Faculty of Commerce \\ Helwan University \\ Egypt
}

2010 Mathematics Subject Classification: 62P30.

Keywords and phrases: quality control, multivariate process, MCUSUM quality control chart, generalized variance $|S|$ quality control chart.

Received November 14, 2017

(C) 2017 Scientific Advances Publishers 


\begin{abstract}
This paper compares the performance of two multivariate methods, based on Multivariate Cumulative Sum (MCUSUM) quality control chart and generalized variance $|S|$ quality control chart. MCUSUM control charts are widely used in industry because they are powerful and easy to use. They cumulate recent process data to quickly detect out-of-control situations. MCUSUM procedures will usually give tighter process control than classical quality control charts. A MCUSUM signal does not mean that the process is producing bad product. Rather it means that action should be taken so that the process does not produce bad product. MCUSUM procedures give an early indication of process change, they are consistent with a management philosophy that encourages doing it right the first time (Pignatiello and Kasunic [25]). MCUSUM charts tend to have inertia that later data points carry with them. As a result, when a trend occurs on one direction of the target mean and a resulting shift occurs in the other direction of the target mean, the two types of charts will not pick up the shift immediately. Generalized variance $|S|$ quality control chart is very powerful way to detect small shifts in the mean vector. The main purpose of this paper, presents an improved the generalized variance $|S|$ quality control chart for multivariate process. Generalized variance chart allow us to simultaneously monitor whether joint variability of two or more related variables is in control. In addition, a control chart commonly requires samples with fixed size be taken at fixed intervals. It is assumed that in both univariate and multivariate control charts, each sample is independent of the previous samples. Thus, this paper decides comparison of MCUSUM and generalized variance $|S|$ multivariate control chart procedure that there is a strong need for an applied work on the practical development and application study by real data.
\end{abstract}

\title{
1. Introduction
}

The Multivariate Cumulative Sum (MCUSUM) control chart is a multivariate quality control chart that has the advantage of detecting small changes in the process mean.

As a multivariate counterpart of the $\bar{X}$ chart, the $\chi^{2}$-chart was first suggested and used by Hotelling [16] in the testing of bombsights. 
It is well known that the $\chi^{2}$-chart is relatively insensitive to small mean shifts. This disadvantage raises the problem of how to obtain multivariate extensions of run rules, the CUSUM charts (Woodall and Ncube [34]) suggested a multiple CUSUM chart by using a series of the CUSUM control charts on original characteristics or on principal component axes depending on the type of shift in the mean that is considered to be important to detect. Pignatiello and Runger [26] showed that the Woodall and Ncube [34] multiple CUSUM chart does not have good average run length (ARL) properties when the process means shifts along several characteristics simultaneously. To lessen the sensitivity of the multiple univariate CUSUM chart to directions, they recommended using the univariate CUSUM charts aimed at several uniformly elected directions. Obviously, the more the directions the less the sensitivity. But, at the same time they found that the resulting control chart is hard to manage when there are three or more characteristics. Hawkins [15] indicated that under some circumstances separate controls on the regression - adjusted variables by the CUSUM charts can both improve the speed of detection and make the chart signal more easily interpretable.

The above problem also motivates attempts to extend the univariate CUSUM statistics to multivariate data. One difficulty encountered with generalizing the CUSUM statistics is that there are two cumulative sums for each variable, see Crosier [9, 10], Pignatiello and Runger [26], proposed some two-sided methods which require only cumulative sum, then they generalized these new statistics to the higher dimension. As a result, they obtained two multivariate CUSUM charts: the MCUSUM and multivariate CUSUM(I) $(\mathrm{MCl})$ charts. Note that neither of these two charts is the natural multivariate extension of the univariate CUSUM chart. 
The rapid growth data acquisition technology and the uses of online computers for process monitoring led to an increased interest in the simultaneous control of several related quality characteristics. These techniques are often referred to multivariate statistical process control procedures. The use of separate univariate control chart for each quality characteristic has proved to be inappropriate. This is because, it neglects the correlation between the multiple quality characteristics; and this leads to incorrect results.

The modern statistical process control took place when Shewhart [28] developed the concept of a control chart based on the monitoring of the process mean level through sample mean ( $\bar{X}$ chart) and process dispersion through sample range ( $R$ chart) or sample standard deviation chart. In the multivariate setting, Hotelling [16] published what can be called the first major works in multivariate quality control. Hotelling developed the $T^{2}$ statistic and the statistics based on the sample variance-covariance matrix $S$ procedure, and its extensions to control charts to combine measurements taken on variables in several dimensions into a single measure of excellence.

After Hotelling there was no significant work done in this field until the early sixties, when with the advances in computers, interest in multivariate statistical quality control was revived. Since then, some authors have done some work in this area of multivariate quality control.

The two more important and used statistics based on the sample variance-covariance matrix $S$, are Likelihood-Ratio and the generalized variance $|S|$, the 3 determinant of $S$, Wilks [32]; Anderson [5]; Korin [21]; Alt [2, 3]; Aparisi et al. [6]; Dogu and Kocakoc [12].

Alt [2] proposed several control charts based on the sample generalized variance, denoted by $|S|$. One of these methods using the $|S|$ control chart is to utilize its distributional properties. The other method 
is constructed using the first two moments of the $|S|$ and the property that most of the probability distribution of $|S|$ is contained in the interval: $E(|S|) \pm 3[V(|S|)]^{\frac{1}{2}}$.

Houshmand and Javaheri [17] presented two procedures to control the covariance matrix in a multivariate setting. The advantages of these procedures are that they allow the investigators to identify the sources of the out-of-control signal. These procedures are based on constructing tolerance regions to control the parameters of the correlation matrix.

Linna et al. [22] presented a model for correlated quality variables with measurement error. The model determined the performance of the multivariate control charting methods. The usual comparison of control chart performance does not directly apply in the presence of measurement error.

Djauhari [11] proposed an improved control chart having unbiased control limits. This method focuses on use of determinate of the average of sample as an estimate of the true generalized variance and its square root. In this paper which refers to the use of $|S|$ and $\sqrt{|S|}$ as multivariate dispersion measures have some limitations. In general, some changes of the covariance structure do not change the sample generalized variance and are not apt to be detected using $|S|$ and $\sqrt{|S|}$ chart. He presented these charts and improved their effectiveness by correcting the base of the control limits. He also discusses the problems encountered when he uses the standard chants. He derived the bias of their control limits and suggests improvements.

\section{Multivariate Quality Control Chart}

Multivariate quality control charts are a type of variables control that how correlated, or dependent, variables jointly affect a process or outcome. The multivariate quality control charts are powerful and simple 
visual tools for determining whether the multivariate process is incontrol or out-of-control. In the other words, control charts can help us to determine whether the process average (center) and process variability (spread) are operating at constant levels. Control charts help us focus problem - solving efforts by distinguishing between common and assignable cause variation. Multivariate control chart plot statistical from more than one related measurement variable. The multivariate control chart shows how several variables jointly influence a process or outcome.

It is demonstrated that if the data include correlated variables the use of separate control chart is misleading because the variables jointly affect the process. If we use separate univariate control chart in a multivariate situation, type I error and probability of a point correctly plotting in-control are not equal to their expected values the distortion of those values increases with the number of measurement variables.

It is shown that multivariate control chart has several advantages in comparison with multiply univariate charts:

- The actual control region of the related variables is represented.

- We can maintain specification type I error.

- A signal control limit determines whether the process is in control.

- Multivariate control chart simultaneously monitors two or more correlated variables. To monitor more than one variable using univariate charts, we need to create a univariate chart for each variable.

- The scale on multivariate control charts unrelated to the scale of any of the variables.

- Out-of-control signals in multivariate charts do not reveal which variable or combination of variables cause the signal. 
A multivariate control chart consists of:

- Plotted points, each for which represents a rational subgroup of data sampled from the process, such as a subgroup mean vector individual observation, or weighted statistic.

- A center line, which represents the expected value of the quality characteristics for all subgroups.

- Upper and lower control limits (UCL and LCL), which are set a distance above and below the center line. These control limits provide a visual display for the expected amount for variation. The control limits are based on the actual behaviour of the process, not the desired behaviour or specification limits. A process can be in control and yet not be capable of meeting requirements.

\section{Comparison Methodology}

\subsection{Univariate cumulative sum (CUSUM) procedure}

Cumulative sum (CUSUM) control charts were first introduced by Page [24]. He proposed that, a decision about changes in a process should not be based on a single observation or a statistic calculated from a few observations, but on all the observations that had been obtained up to the time of testing.

The information from the new sample should be combined with past data to give an indication of a possible shift in the process level. Often successive observations cannot be considered independent, so combining past and present data should increase sensitivity and speed the detection of small shifts in a process.

Montgomery [23] considered that the (CUSUM) control chart is a good alternative when small shifts are important. He proposed that, the CUSUM chart directly incorporates all the information in the sequence of sample values by plotting the cumulative sums of the deviations of the sample values from a target value. 
He supposed the samples of size $n \geq 1$ are collected, and $\bar{X}_{j}$ is the average of the $j$-th sample. Then if $\mu_{0}$ is the target for the process mean, the cumulative sum control is formed by plotting the quantity

$$
C_{i}=\sum_{j=1}^{i}\left(\bar{X}_{j}-\mu_{0}\right)
$$

Against the sample $i, C_{i}$ is called the cumulative sum up to and including the $i$-th sample. Because they combined information from several samples, cumulative charts are more effective than Shewhart charts for detecting small process shifts. He adds that, furthermore, they are particularly effective with samples of size $n=1$.

This makes the cumulative sum control chart a good candidate for use in the chemical and process industries were rational subgroups are frequently of size one, and in discrete parts manufacturing with automatic measurement of each part and on-line control using a microcomputer directly at the work center.

He notes that there are two ways to represent CUSUMS, the tabular (or algorithmic) CUSUM, and the $V$-mask form of the CUSUM.

\subsection{The tabular or algorithmic CUSUM for monitoring the process mean}

Montgomery [23] shown that a tabular CUSUM may be constructed for monitoring the mean of a process. CUSUMs may be constructed both for individual observations and for the averages of rational subgroups. The case of individual observations occurs very often in practice, so that situation will be treated first. Later he shown that how to modify these results for rational subgroups.

Let $X_{i}$ be the $i$-th observation on the process. When the process is in control $X_{i}$ has a normal distribution with mean $\mu_{0}$ and standard deviation $\sigma$ he assumed that either $\sigma$ is known or that an estimate is available. 
Sometimes it is considering that $\mu_{0}$ as a "target" value for the quality characteristic $X$, this viewpoint is often taken in the chemical and process industries when the objective is to control $X$ to a particular target value if the process drifts or shifts off his target value, the CUSUM will signal, and adjustment is made to some manipulatable variable to bring the process back on target. Also, in some cases, a signal from a CUSUM indicates the presence of an assignable cause that must be investigated just as in the Shewhart chart case. The tabular CUSUM works by accumulating derivations from $\mu_{0}$ that are above target with one statistic $C^{+}$and accumulating derivations from $\mu_{0}$ that are below target with another statistic $C^{-}$. The statistics $C^{+}$and $C^{-}$are called one-sided upper and lower CUSUMs, respectively. They are computed as follows:

The tabular CUSUM

$$
\begin{aligned}
& C_{i}^{+}=\max \left[0, X_{i}-\left(\mu_{0}+k\right)+C_{i-1}^{+}\right], \\
& C_{i}^{-}=\max \left[0,\left(\mu_{0}-k\right)-X_{i}+C_{i-1}^{-}\right],
\end{aligned}
$$

where the starting values are $C_{i}^{+}=C_{i}^{-}=0$.

In Equations (2) and (3), $k$ is usually called the reference value (or the allowance, or the slack value), and it is often chosen about halfway between the target $\mu_{0}$ and the out-of-control value of the mean $\mu_{1}$ that it was interested in detecting quickly. Thus, if the shift is expressed in standard deviation units as

$$
\mu_{1}=\mu_{0}+\delta \sigma\left(\text { or } \delta=\frac{\left|\mu_{1}-\mu_{0}\right|}{\sigma}\right)
$$

then $k$ is one-half the magnitude of the shift or

$$
k=\frac{\delta}{2} \sigma=\frac{\left|\mu_{1}-\mu_{0}\right|}{2} .
$$


Note that $C_{i}^{+}$and $C_{i}^{-}$accumulate deviations from the target value $\mu_{0}$ that are greater than $k$, with both quantities reset to zero on becoming negative. If either $C_{i}^{+}$or $C_{i}^{-}$exceed the decision interval $H$, the process is considered to be out-of-control.

The action taken following an out-of-control signal on a CUSUM control scheme is identical to that with any control: one should search for the assignable cause, take any corrective action required, and then reinitialize the CUSUM at zero. The CUSUM is particularly helpful in determining when the assignable cause has occurred just count backward from the out of control signal to the time period when the CUSUM lifted above zero to find the first period following the process shift. The counters $N^{+}$and $N^{-}$are used in this capacity $\left(N^{+}\right.$and $N^{-}$indicate the number of consecutive periods that the CUSUM $C_{i}^{+}$or $C_{i}^{-}$have been nonzero).

In situations where an adjustment to some manipulatable variable is in order to bring the process back to target value $\mu_{0}$, it may be helpful to have an estimate of the new process mean following the shift. This can be computed as follows:

$$
\hat{\mu}= \begin{cases}\mu_{0}+k+\frac{C_{i}^{+}}{N^{+}}, & \text {if } C_{i}^{+}>H, \\ \mu_{0}-k-\frac{C_{i}^{-}}{N^{-}}, & \text {if } C_{i}^{-}>H .\end{cases}
$$

Finally, Montgomery [23] noted that runs tests, and other sensitizing rules such as the zone rules, cannot be safely applied to the CUSUM, because successive values of $C_{i}^{+}$and $C_{i}^{-}$are not independent. In fact, the CUSUM can be thought of as a weighted average. Where the weights are stochastic or random. 
Now, it is necessary to summarize the recommendations for CUSUM design as follows:

(1) The tabular CUSUM is designed by choosing values for the reference value $k$ and the decision interval $H$.

(2) These parameters can be selected to provide good average run length performance.

(3) There have been many analytical studies of CUSUM ARL performance. On which, we can select values of $H$ and $k$.

(4) Several techniques can be used to calculate the ARL of a CUSUM. Vance [31] provides a very accurate computer program. A number of authors have used an approach to calculating ARLs due to Brook and Evans [8] that is based on approximating transitions from the in-control to the out-of-control state with a Markov chain Howkins [14] has provided a simple but very accurate ARL calculation procedure based on an approximating equation. His approximation requires a table of constants to apply and is accurate to within 1-3\% of the true ARL value. Woodall and Adams [33] recommend the ARL approximation given by Siegmund [29] because of its simplicity.

For a one-sided CUSUM (that is, $C_{i}^{+}$or $C_{i}$ ) with parameters $h$ and $k$, Siegmund's approximation is,

$$
A R L=\frac{\exp (-2 \Delta b)+2 \Delta b-1}{2 \Delta^{2}}, \quad \text { for } \Delta \neq 0,
$$

where

$$
\begin{gathered}
\Delta=\left\{\begin{array}{l}
\delta^{*}-k \text { for the upper one-sided CUSUM } C_{i}^{+}, \\
-\delta^{*}-k \text { for the lower one-sided CUSUM } C_{i}^{-},
\end{array}\right. \\
b=h+1.166 \text { and } \delta^{*}=\left(\mu_{1}-\mu_{0}\right) / \sigma .
\end{gathered}
$$

If $\Delta=0$, one can use $A R L=b^{2}$. 
The quantity $\delta^{*}$ represents the shift in the mean, in the units of $\sigma$, for which the ARL is to be calculated. Therefore, if $\delta^{*}=0$, we would calculate $A R L_{0}$ from Equation (6) whereas if $\delta^{*} \neq 0$, we would calculate the value of ARLs of the two one-sided statistics - say, $A R L^{-}$by using:

$$
\frac{1}{A R L}=\frac{1}{A R L^{+}}+\frac{1}{A R L^{-}}
$$

Generally, $k$ is choosing relative to the size of the shift we want to detect: that $k=\frac{1}{2} \delta$, where $\delta$ is the size of the shift in standard deviation units. This approach comes very close to minimizing the $A R L_{1}$ value for detecting a shift of size $\delta$ for fixed $A R L_{0}$. It is necessary to note that a widely mentioned earlier, a widely used value of $k$ in practice

is $k=\frac{1}{2}$. Then, once $k$ is selected you should choose $h$ to give the desired in-control $A R L_{0}$ performance.

\section{The standardized CUSUM}

Many users the CUSUM prefer to standardize the variable $X_{i}$ before performing the calculations. Let

$$
y_{i}=\frac{x_{i}-\mu_{0}}{\sigma}
$$

be the standardized value of $X_{i}$. Then the standardized CUSUMs are defined as follows.

The standardized two-sided CUSUM

$$
\begin{aligned}
C_{i}^{+} & =\max \left[0, y_{i}-k+C_{i-1}^{+}\right], \\
C_{i}^{-} & =\max \left[0,-k-y_{i}+C_{i-1}^{-}\right] .
\end{aligned}
$$


Montgomery [23] noted that, there are two advantages to standardizing the CUSUM. First, many CUSUM charts can be have the same values of $k$ and $h$, and the choices of these parameters are not scale dependent (that is, they do not depend on $\sigma$ ). Second, a standardized CUSUM leads naturally to a CUSUM for controlling variability.

\subsection{The $V$-mask procedure}

Montgomery shown that the $V$-mask is an alternative procedure to the use of a tabular CUSUM is the $V$-mask control scheme proposed by Barnard [7]. The $V$-mask is applied to successive values of the CUSUM statistic.

$$
\begin{aligned}
C_{i} & =\sum_{j=1}^{i} y_{i} \\
& =y_{i}+C_{i-1},
\end{aligned}
$$

where $y_{i}$ is the standardized observation $y_{i}=\frac{\left(X_{i}-\mu_{0}\right)}{\sigma}$. A typical $V$-mask is shown in Figure 1.

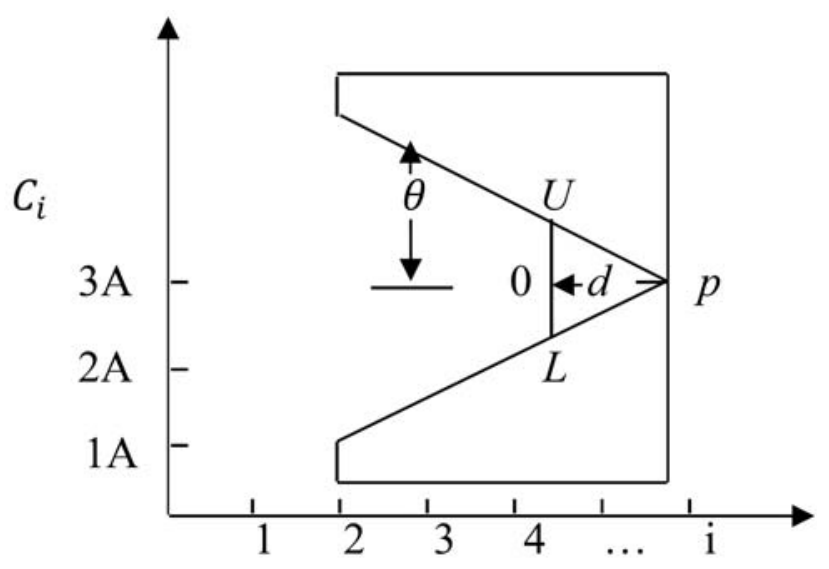

Figure 1. A typical $V$-mask. 
The decision procedure consists of placing the $V$-mask on the cumulative sum control chart with the point (0) on the last value of $C_{i}$ and the line $0 P$ parallel to the horizontal axis. If all the previous cumulative sums, $C_{1} C_{2} \ldots C_{i}$ lie within the two arms of the $V$-mask, the process is in control. However, if any of the cumulative sums lie out-side the arms of the mask, the process is considered to be out-of-control. In actual use, the $V$-mask would be applied to each new point on the CUSUM chart as soon as it was plotted, and the arms are assumed to extend backward to the origin. The performance of the $V$-mask is determined by the lead distance $d$ and the angle $(\theta)$ shown in Figure 1.

The tabular CUSUM and the $V$-mask scheme are equivalent if

$$
k=A \tan \theta,
$$

and

$$
h=A d \tan (\theta)=d k .
$$

In these two equations, $A$ is the horizontal distance on the $V$-mask plot between successive points in terms of unit distance on the vertical scale where $k=\frac{1}{2}$ and $h=5$ we would select $A=1$ (say), and then Equations (11) and (12) would be solved as follows:

$$
\begin{aligned}
k & =A \tan \theta, \\
\frac{1}{2} & =(1) \tan \theta,
\end{aligned}
$$

or

$$
\theta=26.57^{\circ}
$$

and

$$
h=\mathrm{d} k,
$$




$$
5=d\left(\frac{1}{2}\right)
$$

or

$$
d=10 .
$$

That is the lead distance of the $V$-mask would be 10 horizontal positions, and the angle opening on the $V$-mask would be $26.57^{\circ}$.

Johnson [20] has suggested a method for designing the $V$-mask, that is, selecting $d$ and $\theta$. He recommended the $V$-mask parameters.

$$
\theta=\tan ^{-1}\left(\frac{8}{2 A}\right)
$$

and

$$
d=\left(\frac{2}{\delta^{2}}\right) \ln \left(\frac{1-\beta}{\alpha}\right)
$$

where $2 \alpha$ is the greatest allowable probability of a signal when the process mean is on target (a false alarm) and $\beta$ is the probability of not detecting a shift of size $\delta$. If $\beta$ is small, which is usually the case, then.

$$
d \approx-2\left(\frac{\ln (\alpha)}{\delta}\right)
$$

Montgomery [23] strongly advise the quality engineer not to use the $V$-mask procedure. Some of the disadvantages and problems associated with this scheme are as follows:

(1) The $V$-mask is a two-sided scheme: it is not very useful for onesided process monitoring problems.

(2) The head start feature, which is very useful in practice, cannot be implemented with the $V$-mask. 
(3) It is sometimes difficult to determine how far backwards the arms of the $V$-mask should extend thereby making interpretation difficult for the practitioner.

(4) Perhaps the biggest problem with the $V$-mask is the ambiguity associated with $\alpha$ and $\beta$.

Adams et al. [1] point out that detaining $2 \alpha$ as the probability of a false alarm is incorrect. Essentially $2 \alpha$ cannot be the probability of a false alarm on any single sample because this probability changes over time on the CUSUM nor can $2 \alpha$ be the probability of eventually obtaining a false alarm (this probability is, of course, l). In fact, $2 \alpha$ must be the long-run proportion of observations reservations resulting in false alarms. If this is so, then the in-control ARL should be $A R L_{0}=\frac{1}{2 \alpha}$. However, Johnson's design method produces values of $A R L_{0}$ that are substantially larger than $\frac{1}{2 \alpha}$.

Note that the actual values of $A R L_{0}$ are about five times the desired value used in the design procedure. The schemes will also be much less sensitive to shifts in the process mean. Consequently, the use of the $V$-mask scheme is not a good idea unfortunately, it's still the default CUSUM in many SPC software packages. Hopefully computer software developers will soon replace this procedure with the algorithmic or tabular CUSUM.

Alwan [4] presents a CUSUM control scheme based on the sequential probability ration test. He defines the statistic $E_{i}$ as

$$
E_{i}=M\left(\bar{X}_{i}-\mu_{0}\right)^{\backslash \Sigma^{-1}}\left(\bar{X}_{i}-\mu_{0}\right)
$$

and indicates that $E_{i}$ is distributed as a noncentral $\chi^{2}$ distribution. Then, he proposes a sequential test in reverse order on the noncentrality 
parameter of the distribution of statistic $E_{i}$ Alwan [4] shown that the decision equation of the test is linear and therefore a standard $V$-mask can be constructed.

\subsection{The multiple univariate CUSUM control charts}

Woodall and Ncube [34] introduced the use of a one-sided or two sided univariate CUSUM chart to monitor a $p$-dimensional multivariate normal process. They assumed that the independent $p$-characteristic random variables $X_{t}, t=1,2, \ldots$ are successive samples from a $p$-dimensional multivariate normal process with mean $\mu_{0}$ and variancecovariance matrix $\Sigma$. The run length of a one-sided procedure for detecting the positive mean shift of the $j$-th characteristic is:

$$
N(j)=\operatorname{MIN}\left\{t: S_{j, t} \geq H_{j}\right\},
$$

where

$$
S_{j, t}=\operatorname{Max}\left\{0, S_{j, t-1}+X_{j, t}-k_{j}\right\}, t=1,2, \ldots
$$

In Equation (16), $X_{j, t}$ refers to the $j$-th characteristic of the $t$-th observation, $S_{j, t}$ is the upper CUSUM of the $j$-th, characteristic after $t$ observations, $H_{j}>0$ is the upper decision interval or control limit for characteristic $j$ and $k_{j}$ is the reference value for characteristic $j$. The choice of the reference value $k_{j}$ depends on the shift in the mean that is considered to be important and needs to be detected quickly for the $j$-th characteristic.

To detect shifts in either direction, the run length of the two-sided CUSUM procedure is defined as:

$$
N(j)=\operatorname{MIN}\left\{t: S_{j, t} \geq H_{j} \text { or } T_{j, t} \leq-H_{j}\right\}
$$


where

$$
\begin{gathered}
T_{j, t}=\operatorname{MIN}\left\{0, T_{j, t-1}+X_{j, t}+K_{j}\right\} \\
t=1,2, \ldots, H_{j}<T_{j, 0} \leq 0
\end{gathered}
$$

and $T_{j, t}$ is the lower CUSUM of the $j$-th characteristic after $t$-th observations. The run length of the multiple CUSUM procedure is defined as,

$$
N=\operatorname{MIN}\{N(1), N(2), \ldots, N(p)\}
$$

Therefore, the process is considered out of control as soon as any one of the multiple CUSUM control charts indicates an out of control signal. This method has two obvious advantages. It is very easy to understand and very easy to implement.

However, it has a major disadvantage in that the correlation between the various quality characteristics is not taken into account. Therefore, it is impossible to tell exactly what is the significance level of the test.

\subsection{Multivariate CUSUM charts}

Pignatiello and Runger [26] introduced two multivariate CUSUM charting procedures. Both these multivariate CUSUM procedures are based on quadratic forms of the mean vector. The difference between the two multivariate CUSUM procedures discussed here centers on the point at which the accumulation (i.e., the sum) is made. Multivariate CUSUM (I) (MC1) accumulates the $X$ vectors before producing the quadratic forms while multivariate CUSUM (II) (MC2) calculates a quadratic form for each $X$ and then accumulates those quadratic forms. A procedure similar to $\mathrm{MC} 1$, described below, was first proposed by Pignatiello and Kasunic [25] in the following sections. 


\subsubsection{Multivariate CUSUM (I)}

To introduce the first multivariate CUSUM charts, they considered that the multivariate sum

$$
C_{t}=\sum_{i=t-n_{t}+1}^{t}\left(X_{i}, \mu_{0}\right)
$$

where $n_{t}$ is formally defined in (17) and can be interpreted as the number of subgroups since the most recent renewal (i.e., zero value) of CUSUM. Since $\frac{1}{n_{t}} C_{t}$ may be written as

$$
\frac{1}{n_{t}} C_{t}=\left(\frac{1}{n_{t}} \sum_{i=t-n_{t}+1}^{t} X_{i}\right)-\mu_{0}
$$

The vector $\frac{1}{n_{t}} C_{t}$ represents the difference between the accumulated sample average and the target value for the mean. Consequently, at time $t$, the multivariate process mean can be estimated to be $\frac{1}{n_{t}} C_{t}+\mu_{0}$. The norm of $C_{t}$

$$
\left\|C_{t}\right\|=\sqrt{C_{t}^{\backslash} \Sigma^{-1} C_{t}}
$$

is seen as a measure of the distance of our estimate of the mean of the process from the target mean for the process. A multivariate control chart can be constructed by defining MC1 as,

$$
M C 1_{t}=\max \left\{\left\|C_{t}\right\|-k n_{t}, 0\right\},
$$

and

$$
n_{t}=\left\{\begin{array}{cl}
n_{t-1}+1 & \text { if } M C 1_{t-1}>0 \\
1 & \text { otherwise }
\end{array}\right.
$$


where the choice of the reference value $k>0$ is discussed below. The MC1 chart operates by plotting $M C 1_{t}$ on a control chart with an upper control limit of $U C L_{1}$ if $M C 1_{t}$ exceeds $U C L_{1}$ then the process is deemed to be off-target.

Because the MC1 chart can not be modeled as a simple stationary Markov chain, Pignatiello and Runger [26] use a Montocarlo simulation to evaluate the ARL performance of the chart.

\subsubsection{Multivariate CUSUM (II)}

Pignatiello and Runger [26] proposed a method denoted by MC2, based on the square of distance of each sample mean from $\mu_{0}$ and accumulate those squared distances they defined the square distance of the $t$-th sample mean from the target value of $\mu_{0}$, where

$$
D_{t}^{2}=\left(X_{t}-\mu_{0}\right)^{\backslash \Sigma^{-1}}\left(X_{t}-\mu_{0}\right)
$$

has $\chi^{2}$ distribution with $p$ degrees of freedom when the process is ontarget and a non-central $\chi^{2}$ distribution when the process is off-target. A one-sided univariate CUSUM can now be formed as

$$
M C 2_{t}=\max \left\{0, M C 2_{t-1}+D_{t}^{2}-k\right\}
$$

with $M C 2_{0}=0$. The primary difference in the two CUSUM charts is that MC1 accumulates the sample mean vector prior to the production of the quadratic forms, while MC2 calculates the quadratic forms for each sample mean and then accumulates the values of those forms.

Moreover, Pignatiello and Runger [26] compared MC1 and MC2 to the multiple univariate CUSUM charts given by Woodall and Ncube [34] and to the multivariate Shewhart $\chi^{2}$ charts. The results show that the ARL of the MC1 chart outperforms the other three charts in almost all cases. Crosier [10] also presented two multivariate cumulative sum (MCUSUM) quality control charts. 
The first CUSUM chart reduces each observation vector or sample mean vector to a $t$-statistic (the square root of the right-hand side of Equation (17)) and then forms a CUSUM of the $T$-statistics. Crosier states that a problem with this method is that when a shift of the mean is indicated, the procedure gives no indication of where the shift occurs.

The second method derived by crosier is a two-sided vector-value CUCUM scheme. He shrinks the updated CUSUM toward zero after each observation. The shrinkage is performed by multiplication rather than by addition or subtraction. Crosier defined the statistic $C_{i}$ the CUSUM after the $i$-th sample mean vector as,

$$
C_{i}=\left\{\left(S_{i-1}+X_{i}\right)^{\backslash \Sigma^{-1}}\left(S_{i-1}+X_{i}\right)\right\}^{\frac{1}{2}}
$$

where

$$
S_{i}= \begin{cases}0 & \text { if } C_{i} \leq k_{1}, \\ \left(S_{i-1}+X_{i}\right)\left(1-\frac{k_{1}}{C_{i}}\right) & \text { if } C_{i}>k_{1},\end{cases}
$$

and $i=1,2, \ldots$; where $S_{0}=0$ and $k_{1}>0$.

Note that $k_{1}>0$ is the reference value of the scheme and $C_{i}$ is the generalized length of the CUSUM vector before shrinking.

Multivariate CUSUM scheme, denoted by MCUSUM, signals when

$$
Y_{i}=\left\{S_{i}^{\backslash \Sigma^{-1}} S_{i}\right\}^{\frac{1}{2}}>h_{2}
$$

where $h_{2}>0$, and $h_{2}$ is the decision interval. The multivariate CUSUM chart independently proposed and developed by Crosier [10] is similar to, yet distinctively different from Pignatiello and Runger [26] MC1, CUSUM procedure. Crosier [10] "contracts" or "shrinks" each of his vectors of cumulative sums toward the zero vector by multiplying the cumulative sum by a scalar. The norm of the contracted cumulative sum 
is then compared with an upper control limit. It turns out that the ARL performance of crosier's multivariate cumulative sum chart is similar to that Pignatiello and Runger [26] MC1 chart. Smith [30] developed another multivariate CUSUM procedure based on the likelihood ratio test. She also extended the procedure to study shifts in the covariance matrix of a multivariate Normal process and to study shifts in the probability of multinomial process compared the statistical performance of the procedure with Alt and Smith [3], Crosier [10] and Pignatiello's method under the bivariate case using simulation.

\subsection{Multivariate process variability control chart}

Monitoring process variability is an important part of any control procedure. Montgomery [23] point out that "just as it is monitor process variability". A similar remark is made by Alt and Smith [3]. Events that need to take place for a successful important of statistical process control include an initial examination of stability and a capability analysis. Once this stage is satisfactorily completed, ongoing monitoring of key process parameters is necessary. One vital parameter is process variability, and the need to monitor and control variability motivates this paper.

It focuses on use of the determinate of the sample variancecovariance matrix $|S|$, also called the sample generalized variance.

Jackson [18] proposed that the starting point of the statistical application of the method of principal components is the sample covariance matrix $S$, for a $p$-variate problem,

$$
S=\left[\begin{array}{cccc}
S_{1}^{2} & S_{12} & \ldots & S_{1 p} \\
S_{21} & S_{2}^{2} & \ldots & S_{2 p} \\
\vdots & \vdots & \ddots & \vdots \\
S_{p 1} & S_{p 2} & \ldots & S_{p}^{2}
\end{array}\right],
$$

where $S_{i}^{2}$ is the variance of the $i$-th variable and $S_{i j}$ is the covariance between the $i$-th and $j$-th variables. 
Process variability is summarized by the $p \times p$ covariance matrix. The main diagonal elements of this matrix are the variance of the individual process variability, and the off-diagonal elements are the covariances. If the covariances are not equal to zero, it indicates a relationship existing between those two variables, the strength of that relationship (if it is linear) being represented by the correlation: $r_{i j}=\frac{S_{i j}}{\left(S_{i} S_{j}\right)}$.

Alt and Smith [3] presented two useful procedures variability:

The first procedure is a direct extension of the univariate $S^{2}$ control chart, where $S^{2}$ is the sample variance. The procedure is equivalent to repeated tests of significance of the hypothesis that the process covariance matrix is equal to particular matrix of constants $\sum$. If this approach is used, the statistic plotted on the control chart for the $i$-th sample is

$$
W_{i}=-p n+p n \ln (n)-n \ln \left(\frac{A_{i}}{|\Sigma|}\right)+\operatorname{tr}\left(\Sigma^{-1} A_{i}\right)
$$

where $A_{i}=(n-1) S_{i} . S_{i}$ is the sample covariance matrix for sample $i$, and $t r$ is the trace operator (the trace of matrix is the sum of the main diagonal elements) if the value of $W_{i}$ plots above the upper control limit $U C L=\chi_{\alpha \cdot \frac{p(p+1)}{2}}^{2}$, the process is out-of-control.

The second procedure is based on the sample generalized variances, $|S|$, this statistic, which is the determinant of the sample covariance matrix, is a widely used measure of multivariate dispersion. 
The $|S|$ chart, as presented by Montgomery [23] have dependent on the mean variance of $|S|$ - that is, $E(|S|)$ and $V(|S|)$ - and the property that most of the probability distribution of $|S|$ is contained in the interval: $E(|S|) \pm 3 \sqrt{V(|S|)}$ as follows:

and

$$
\left.\begin{array}{c}
E(|S|)=b_{1}|\Sigma| \\
V(|S|)=b_{2}|\Sigma|^{2}
\end{array}\right\},
$$

where

$$
b_{1}=\frac{1}{(n-1)^{p}} \prod_{i=1}^{p}(n-i)
$$

and

$$
b_{2}=\frac{1}{(n-1)^{2 p}} \prod_{i=1}^{p}(n-i)\left[\prod_{j=1}^{p}(n-j+2)-\prod_{j=1}^{p}(n-j)\right]
$$

Therefore, the parameters of the control chart for $|S|$ would be

$$
\left.\begin{array}{c}
U C L=|\Sigma|\left(b_{1}+3 \sqrt{b_{2}}\right) \\
C L=b_{1}|\Sigma| \\
L C L=|\Sigma|\left(b_{1}-3 \sqrt{b_{2}}\right)
\end{array}\right\} .
$$

The lower control limit in Equation (27) can replaced with zero if the calculated value is less than zero. He noted that, usually in practice $\Sigma$ could be estimate by a sample covariance matrix $S$, based on the analysis of preliminary samples.

Accordingly, we should replace $|\Sigma|$ in Equation (27) by $\frac{|\Sigma|}{b_{1}}$. Since Equation (26) has shown that $\frac{|S|}{b_{1}}$ is an unbiased estimator of $|\Sigma|$. 
As a result, we find that the control chart parameters are

$$
\left.\begin{array}{c}
U C L=|S|\left(1-\frac{3 \sqrt{b_{2}}}{b_{1}}\right) \\
C L=|S| \\
L C L=|S|\left(1-\frac{3 \sqrt{b_{2}}}{b_{1}}\right)
\end{array}\right\} .
$$

\section{The Application}

Delta Fertilizers and Chemical Industries is considered one of the leading companies in the field of fertilizers production in Egypt. About 4500 employees are working for it, on the various managerial levels. Urea production is one of the major products of the company. The production of urea occurs through three stages, summarized as follows:

\section{A. High pressure stage}

In this stage, urea is produced through two reactions; the first reaction occurs by condensation of Ammonia Gas and Carbon dioxide under high pressure and temperature for the sake of the production of intermediate material, known as Carbamate. The second reaction happens by separating the water from the Carbamate in order to a chive urea. In this, stage the condensation of urea approximately $56 \%$. 
It contains 16 variables, these are:

$\begin{array}{lll}1 & \text { X1 } & \begin{array}{l}\text { E-201 Outlet Temperature } \\ 2\end{array} \\ 3 & \text { X2 } & \begin{array}{l}\text { Outlet cold NH3 from E-201 } \\ \text { X3 }\end{array} \\ 4 & \text { X4 } & \text { CO }_{2} \text { to Train } \\ 5 & \text { X5 } & \text { CO }_{2} \text { after E-22 } \\ 6 & \text { X6 } & \text { R-201 } \\ 7 & \text { X7 } & \text { Temperature in reactor R-201 } \\ 8 & \text { X8 } & \text { Temperature in reactor R-201 } \\ 9 & \text { X9 } & \text { Temperature in reactor R-201 } \\ 10 & \text { X10 } & \text { Temperature in reactor R-201 } \\ 11 & \text { X11 } & \text { Stripper level } \\ 12 & \text { X12 } & \text { Liquid leaving the Stripper } \\ 13 & \text { X13 } & \text { Stream from E-204 to j-201 } \\ 14 & \text { X14 } & \text { Conditioned water to Scrubber E-204 } \\ 15 & \text { X15 } & \text { Conditioned water from scrubber E-204 } \\ 16 & \text { X16 } & \text { Stream from j-203 }\end{array}$

Table analysis of laboratory in this stage:

$\begin{array}{cccc}1 & \mathrm{t} 1.1 & \mathrm{NH}_{3} & \text { Reactor outlet } \\ 2 & \mathrm{t} 1.2 & \mathrm{CO}_{2} & \text { Reactor outlet } \\ 3 & \mathrm{t} 1.3 & \mathrm{UR} & \text { Reactor outlet } \\ 4 & \mathrm{t} 1.4 & \mathrm{~B}_{1} & \text { Reactor outlet } \\ 5 & \mathrm{t} 1.5 & \mathrm{H}_{2} \mathrm{O} & \text { Reactor outlet } \\ 6 & \mathrm{t} 2.1 & \mathrm{NH}_{3} & \text { Stripper outlet } \\ 7 & \mathrm{t} 2.2 & \mathrm{CO}_{2} & \text { Stripper outlet } \\ 8 & \mathrm{t} 2.3 & \mathrm{UR} & \text { Stripper outlet } \\ 9 & \mathrm{t} 2.4 & \mathrm{~B}_{1} & \text { Stripper outlet } \\ 10 & \mathrm{~T} 2.5 & \mathrm{H}_{2} \mathrm{O} & \text { Stripper outlet }\end{array}$




\section{B. Low pressure stage}

In this stage, the condensation of urea liquid rises from $56 \%$ to $71 \%$. This happens through the decomposition of the remaining Carbamate and the elimination of water under low pressure.

It contains seven variables, these are:

$\begin{array}{lll}1 & \text { y1 } & \text { Urea solution from stripper E-202 } \\ 2 & \text { y2 } & \text { Steam to E-205 } \\ 3 & \text { y3 } & \text { Urea carbonate solution from stripper T-201 to E-205 } \\ 4 & \text { y4 } & \text { Gas leaving T-201 } \\ 5 & \text { y5 } & \text { Level in TK-201 } \\ 6 & \text { y6 } & \text { P-203 } \\ 7 & \text { y7 } & \text { Urea solution in TK-201 }\end{array}$

Table analysis of laboratory in this stage:

$\begin{array}{llll}1 & \mathrm{t} 3.1 & \mathrm{NH}_{3} & \text { D 202 Outlet } \\ 2 & \mathrm{t} 3.2 & \mathrm{CO}_{2} & \text { D 202 Outlet } \\ 3 & \mathrm{t} 3.3 & \mathrm{UR} & \text { D 202 Outlet } \\ 4 & \mathrm{t} 3.4 & \mathrm{~B}_{1} & \text { D 202 Outlet } \\ 5 & \mathrm{t} 3.5 & \mathrm{H}_{2} \mathrm{O} & \text { D 202 Outlet } \\ 6 & \mathrm{t} 4.1 & \mathrm{NH}_{3} & \text { In TK 201 } \\ 7 & \mathrm{t} 4.2 & \mathrm{CO}_{2} & \text { In TK 201 } \\ 8 & \mathrm{t} 4.3 & \mathrm{UR} & \text { In TK 201 } \\ 9 & \mathrm{t} 4.4 & \mathrm{~B}_{1} & \text { In TK } 201 \\ 10 & \mathrm{t} 4.5 & \mathrm{H}_{2} \mathrm{O} & \text { In TK } 201 \\ 11 & \mathrm{t} 5.1 & \mathrm{NH}_{3} & \text { In PI 302 } \\ 12 & \mathrm{t} 5.2 & \mathrm{CO}_{2} & \text { In PI 302 } \\ 13 & \mathrm{t} 5.3 & \mathrm{UR} & \text { In PI 302 }\end{array}$




\section{Evaporation and prilling stage}

This stage occurs by two stages:

\section{(i) Evaporation stage}

In this stage, the condensation of urea rises from $71 \%$ to $98.7 \%$ approximately and the urea liquid trams forms to urea melt. This happens under high pressure and temperature.

\section{(ii) Prilling stage}

In this stage, the urea melt is through formed into prilling in the prilling tower.

It contains four variables, these are:

$\begin{array}{lll}1 & \text { Z1 } & \text { Urea solution from D-204to E-209 } \\ 2 & \text { Z2 } & \text { D-205 Vacuum } \\ 3 & \text { Z3 } & \text { Urea to prilling tower X-202 } \\ 4 & \text { Z4 } & \text { E-211 Vacuum }\end{array}$

Table analysis of laboratory in this stage:

$\begin{array}{lll}1 & \mathrm{t} 6.1 & \mathrm{~B} 1 \\ 2 & \mathrm{t} 6.2 & \mathrm{H}_{2} \mathrm{O} \\ 3 & \mathrm{t} 6.3 & \text { Pills }>3.35 \\ 4 & \mathrm{t} 6.4 & \text { Pills } 3.35: 2.4 \\ 5 & \mathrm{t} 6.5 & \text { Pills } 2.4: 1.4 \\ 6 & \mathrm{t} 6.6 & \text { Pills } 1.4: 1.0 \\ 7 & \mathrm{t} 6.7 & \text { Pills }<1.0 \\ 8 & \mathrm{t} 6.8 & \mathrm{UR}\end{array}$

\section{Data description}

For the application of multivariate quality control, chart data originate from urea production process, which consists of the three stages and the analysis of laboratory, which discussed above.

The number of the sample is 732 observations taken per hour. 
The advantages of this sample that, it has several variables and several stages of the production. This advantage of the production is the basic reason for choosing this production to allow us to study the multivariate quality control charts.

In this application, we shall introduce the most common using technique of multivariate quality control charts; MCUSUM chart \& generalized variance chart.

\subsection{MCUSUM chart}

- Plotted points, each of which represents the multivariate statistic for each observation.

- A center line (green), which is the median of the theoretical distribution of multivariate statistic.

- Control limits (red), which provide a visual means for assessing whether the process is in-control. The control limits represent the expected variation.

MINITAB marks points outside of the control limits with a red symbol.

\subsubsection{MCUSUM chart of X1, .., X16 and $t 1.1, \ldots, t 2.5$}

Test results for MCUSUM chart of X1, .., X16 and t1.1, .., t2.5.

TEST: One point beyond control limits.

Test Failed at points: (Less than LCL)

$\begin{array}{lllllllllll}112 & 116 & 125 & 130 & 135 & 136 & 139 & 142 & 144 & 146 & 150 \\ 155 & 163 & 165 & 169 & 173 & 174 & 348 & 350 & 352 & 355 & 362 \\ 371 & 375 & 379 & 383 & 386 & 388 & 574 & 579 & 581 & 587 & 591 \\ 592 & 599 & 601 & 603 & 609 & 611 & 617 & 621 & 623 & 625 & 632 \\ 635 & 636 & 643 & 647 & 651 & 653 & 655 & 662 & & & \end{array}$


Test Failed at points: (Greater than UCL)

$\begin{array}{ccccccccccc}11 & 22 & 28 & 42 & 44 & 46 & 49 & 51 & 53 & 55 & 61 \\ 64 & 81 & 102 & 243 & 245 & 251 & 253 & 255 & 258 & 262 & 264 \\ 274 & 449 & 459 & 481 & 634 & 645 & 659 & 659 & 661 & 665 & 701 \\ 715 & 717 & 719 & 722 & 725 & 728 & 731 & & & & \end{array}$

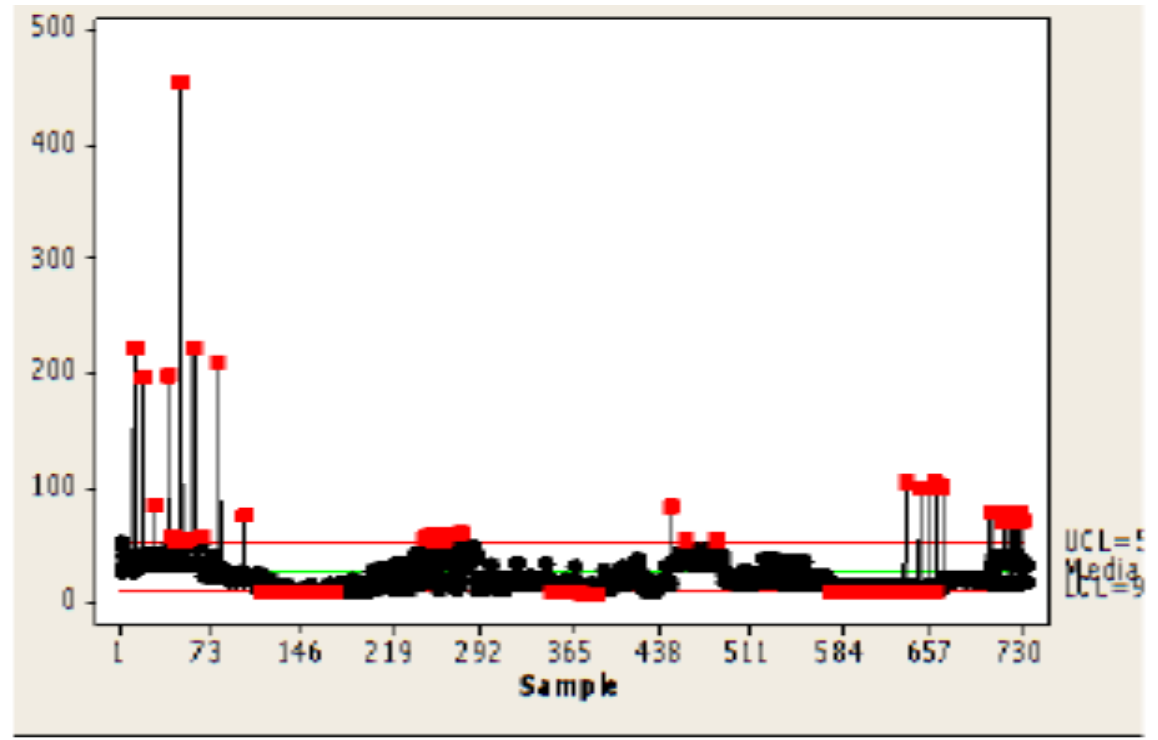

Figure 2. MCUSIM chart of X1, ..., X16 and t1.1, .., t2.5.

MCUSUM chart of X1, .., X16 and t1.1, .., t2.5 can be summarized as follows:

- The lower and upper control limits are 9.7 and 52, respectively. Therefore, we expect the MCUSUM statistics to fall between 9.7 and 52 . The center line or median, is 25.3.

- Test results indicate that 52 points less than LCL, for example, point 114 exceeds the lower control limit.

- Test results indicate that 40 points greater than UCL, for example, the test results indicate that point 13 exceeds the upper control limit. 
- Test results indicate 92 point through beyond the control limits. Then the out-of-control rate $12.6 \%$ and the in-control rate $87.4 \%$.

\subsubsection{MCUSUM chart of $y 1, \ldots, y 7$ and $t 3.1, \ldots, t 4.5$}

Test results for MCUSUM chart of $\mathrm{y} 1, \ldots, \mathrm{y} 7$ and $\mathrm{t} 3.1, \ldots, \mathrm{t} 4.5$.

TEST: One point beyond control limits.

Test Failed at points: (Greater than UCL)

$\begin{array}{llllllllll}30 & 90 & 116 & 132 & 151 & 202 & 251 & 258 & 265 & 269 \\ 552 & 704 & 715 & 717 & 719 & 723 & 725 & 728 & 731 & \end{array}$

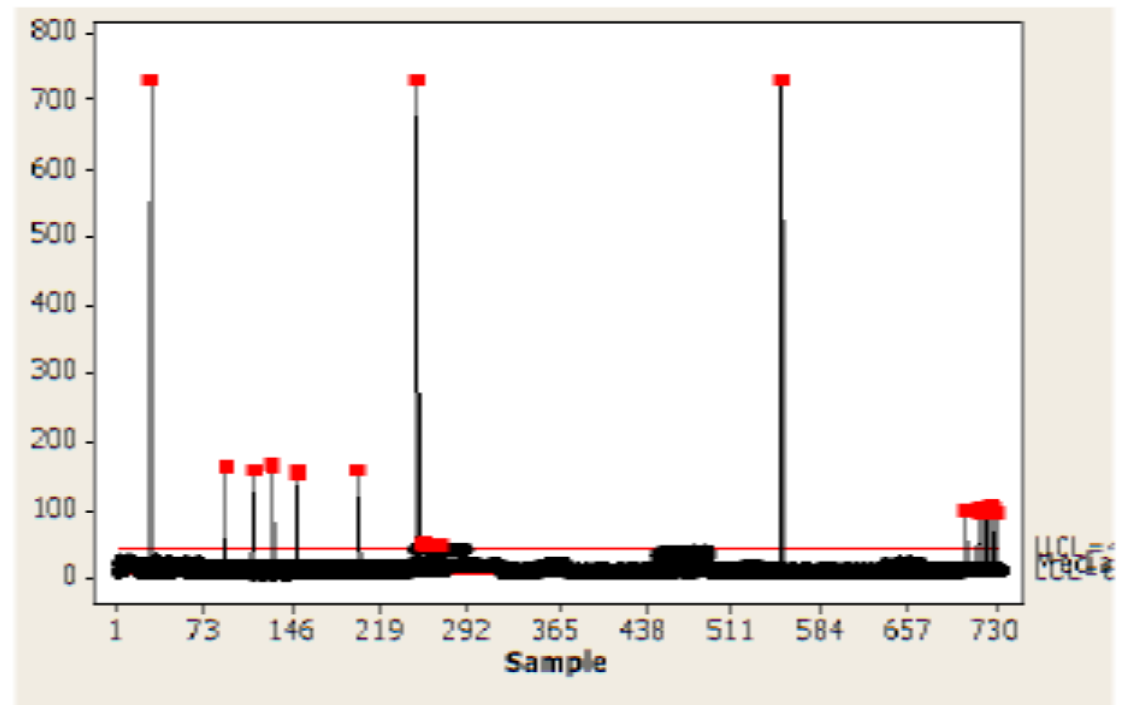

Figure 3. MCUSUM chart of $\mathrm{y} 1, \ldots, \mathrm{y} 7$ and $\mathrm{t} 3.1, \ldots, \mathrm{t} 4.5$.

MCUSUM chart of $y 1, \ldots, y 7$ and $t 3.1, \ldots, t 4.5$ can be summarized as follows:

- The lower and upper control limits are 0.2 and 43.6, respectively. Therefore, we expect the MCUSUM statistics to fall between 0.2 and 43.6. The center line, or median, is 19.3 .

- Test results indicate that 19 point greater than UCL, for example, test results indicate that point 91 exceeds the upper control limit. 
- Test results indicate 19 point that are beyond the control limit. Then the out of control rate $2.59 \%$ and the in-control rate $97.41 \%$.

\subsubsection{MCUSUM chart of $\mathrm{Z1}, \ldots, \mathrm{Z} 4$ and $\mathrm{t6.1}, \ldots, \mathrm{t} 6.8$}

Test results for MCUSUM chart of Z1, .., Z4 and t6.1, ..., t6.8.

TEST: One point beyond control limits.

Test Failed at points: (Greater than UCL)

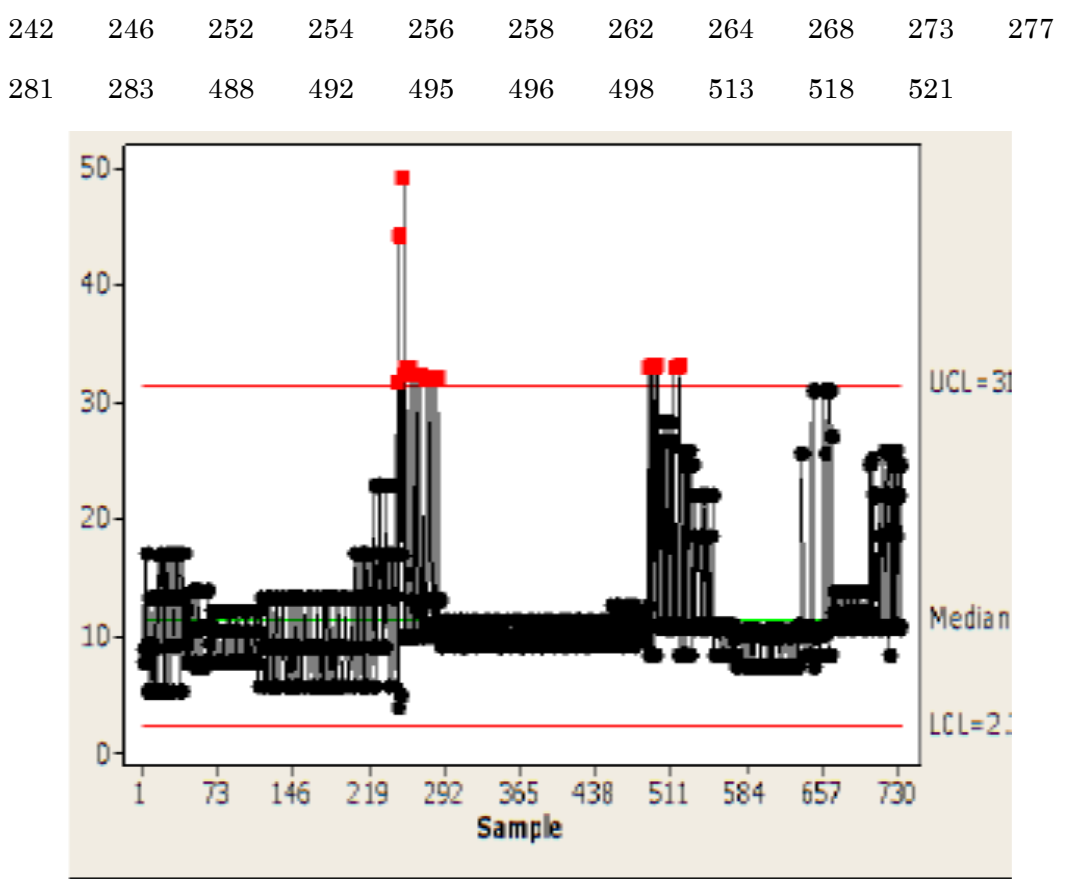

Figure 4. MCUSUM chart of $\mathrm{Z} 1, \ldots, \mathrm{Z} 4$ and $\mathrm{t} 6.1, \ldots, \mathrm{t} 6.8$.

MCUSUM chart of $\mathrm{Z1}, \ldots, \mathrm{Z} 4$ and $\mathrm{t} 6.1, \ldots, \mathrm{t} 6.8$ can be summarized as follows:

- The lower and upper control limits are 2.37 and 31.63, respectively. Therefore, we expect the MCUSUM statistics to fall between 2.37 and 31.63. The center line, or median, is 11.35 .

- Test results indicate that 21 point greater than UCL, for example, test results indicate that point 245 exceeds the upper control limit. 
- Test results indicate 21 point that are beyond the control limit. Then the out of control rate $2.87 \%$ and the in-control rate $97.13 \%$.

\subsection{Generalized variance chart}

A generalized variance chart consists of:

- Plotted points, each of which represents the generalized variance for each observation.

- A center line (green), which is the median of the theoretical distribution of generalized variance statistic.

- Control limits (red), which provide a visual means for assessing whether the process is in-control. The control limits represent the expected variation.

MINITAB marks points outside of the control limits with a red symbol.

\subsubsection{Generalized variance chart of X1, ..., X16 and t1.1, ..., t2.5}

Test results for generalized variance chart of X1, .., X16 and t1.1, ..., t2.5.

TEST: One point beyond control limits.

Test Failed at points:

$\begin{array}{ccccccccccc}11 & 21 & 32 & 41 & 52 & 63 & 82 & 101 & 115 & 119 & 121 \\ 126 & 135 & 136 & 139 & 141 & 145 & 148 & 153 & 155 & 163 & 168 \\ 169 & 170 & 174 & 177 & 181 & 183 & 188 & 195 & 198 & 201 & 208 \\ 222 & 225 & 231 & 248 & 255 & 261 & 265 & 268 & 272 & 276 & 278 \\ 285 & 288 & 394 & 425 & 429 & 433 & 437 & 448 & 449 & 452 & 454 \\ 458 & 459 & 462 & 464 & 466 & 467 & 468 & 469 & 471 & 473 & 475 \\ 476 & 478 & 479 & 481 & 482 & 483 & 484 & 486 & 488 & 489 & 490 \\ 491 & 494 & 513 & 518 & 523 & 526 & 528 & 637 & 647 & 655 & 659 \\ 661 & 665 & 666 & 668 & 671 & 678 & 684 & 688 & 693 & 696 & 698 \\ 702 & 705 & 710 & 713 & 716 & 717 & 718 & 722 & 724 & 726 & 729 \\ 730 & & & & & & & & & & \end{array}$




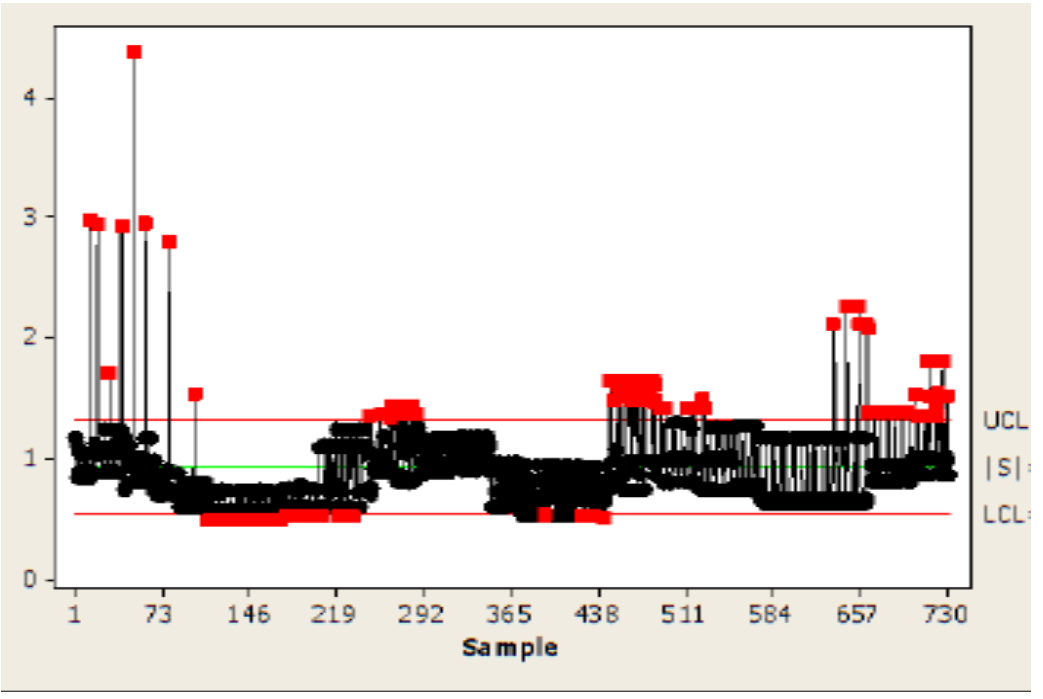

Figure 5. Generalized variance chart of X1, .., X16 and t1.1, .., t2.5.

Generalized variance chart of $\mathrm{X} 1, \ldots, \mathrm{X} 16$ and $\mathrm{t} 1.1, \ldots, \mathrm{t} 2.5$ can be summarized as follows:

- The lower and upper control limits are 0.528 and 1.312, respectively. Therefore, we expect the generalized variance statistics to fall between 0.528 and 1.312. The center line, or median, is 0.92 .

- Test results indicate 111 points through beyond the control limits.

- Test results indicate that the process is in-control for 621 points and out-of-control for 111 points. Then the out-of-control rate $15.16 \%$ and the in-control rate $84.84 \%$. 


\subsubsection{Generalized variance chart of $y 1, \ldots, y 7$ and $t 3.1, \ldots, t 4.5$}

Test results for generalized variance chart of $y 1, \ldots, y 7$ and $t 3.1, \ldots$, $\mathrm{t} 4.5$.

TEST: One point beyond control limits.

Test Failed at points:

$\begin{array}{llllllllll}89 & 112 & 131 & 153 & 202 & 249 & 251 & 255 & 259 & 261 \\ 263 & 266 & 269 & 272 & 275 & 277 & 279 & 280 & 281 & 283 \\ 285 & 286 & 550 & 705 & 713 & 717 & 719 & 723 & 725 & 728 \\ 730 & & & & & & & & & \end{array}$

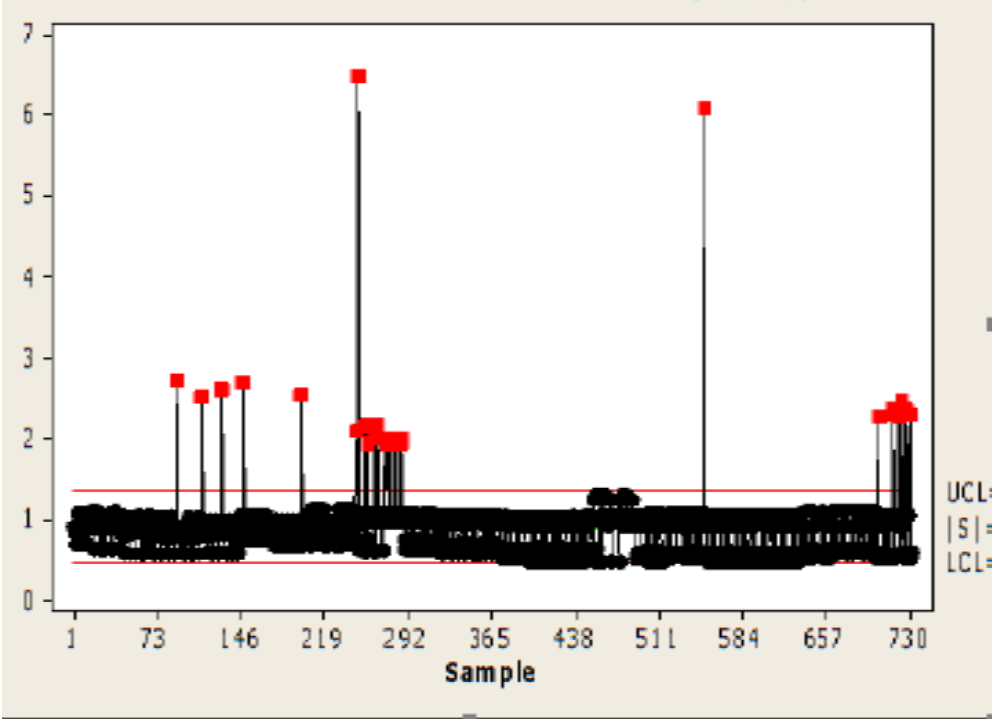

Figure 6. Generalized variance chart of $y 1, \ldots, y 7$ and $t 3.1, \ldots, t 4.5$.

Generalized variance chart of $\mathrm{y} 1, \ldots, \mathrm{y} 7$ and $\mathrm{t} 3.1, \ldots, \mathrm{t} 4.5$ can be summarized as follows:

- The lower and upper control limits are 0.456 and 1.330, respectively. Therefore, we expect the generalized variance statistics to fall between 0.456 and 1.330. The center line, or median, is 0.893 . 
- Test results indicate 31 points through beyond the control limits.

- Test results indicate that the process is in-control for 701 points and out-of-control for 31 points. Then the out-of-control rate $4.23 \%$ and the in-control rate $95.77 \%$.

\subsubsection{Generalized variance chart of $\mathrm{Z} 1, \ldots, \mathrm{Z} 4$ and $\mathrm{t} 6.1, \ldots, \mathrm{t} 6.8$}

Test results for generalized variance chart of $\mathrm{Z1}, \ldots, \mathrm{Z} 4$ and $\mathrm{t6.1}, \ldots$, t6.8.

TEST: One point beyond control limits.

Test Failed at points:

$\begin{array}{llllllllll}243 & 250 & 257 & 263 & 266 & 268 & 272 & 279 & 284 & 288 \\ 489 & 492 & 495 & 497 & 498 & 499 & 502 & 503 & 506 & 509 \\ 510 & 512 & 514 & 516 & 518 & 520 & & & & \end{array}$

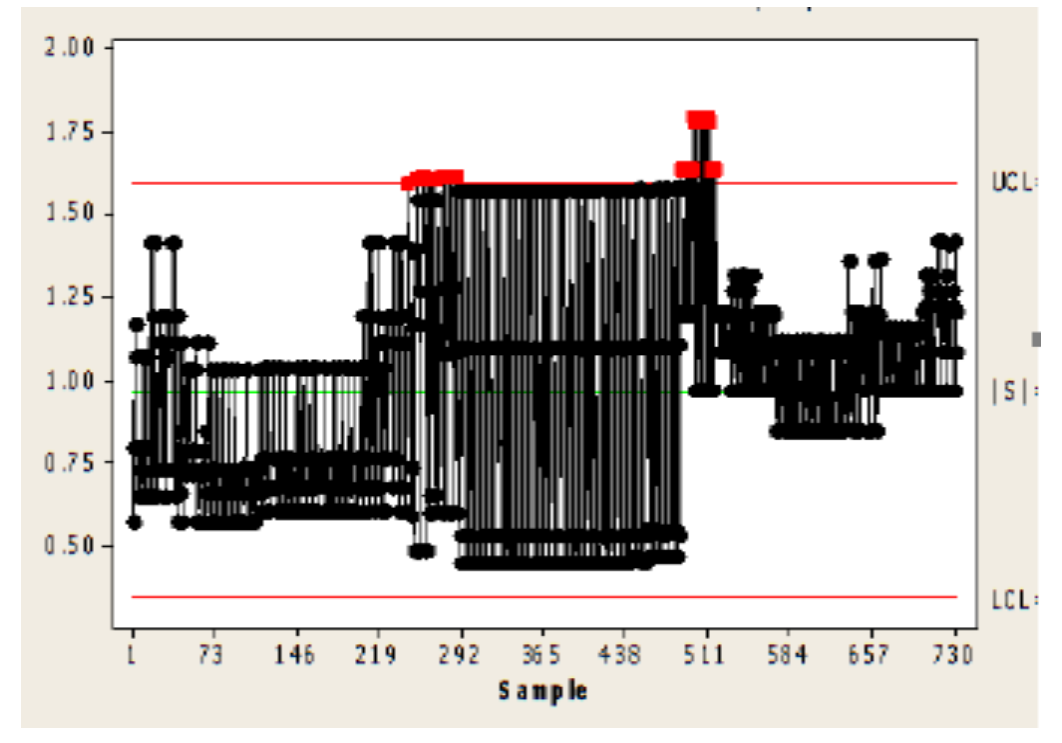

Figure 7. Generalized variance chart of $\mathrm{Z1}, \ldots, \mathrm{Z} 4$ and $\mathrm{t6.1}, \ldots, \mathrm{t} 6.8$. 
Generalized variance chart of $\mathrm{Z} 1, \ldots, \mathrm{Z} 4$ and $\mathrm{t} 6.1, \ldots, \mathrm{t} 6.8$ can be summarized as follows:

- The lower and upper control limits are 0.342 and 1.594, respectively. Therefore, we expect the generalized variance statistics to fall between 0.342 and 1.594. The center line, or median, is 0.968 .

- Test results indicate 26 points through beyond the control limits.

- Test results indicate that the process is in-control for 706 points and out-of-control for 26 points. Then the out-of-control rate $3.55 \%$ and the in-control rate $96.45 \%$.

\section{Comparison Results of Application}

The application is shown that in High process stage, test results of MCUSUM chart indicate that the out-of-control percentage $87.4 \%$ and the in-control percentage $12.6 \%$, and it shown that in Low process stage, test results of MCUSUM chart indicate that the out-of-control percentage $2.59 \%$ and the in-control percentage $97.41 \%$. It is shown that in the Evaporation and Prilling stage, test results of MCUSUM chart indicates that the out-of-control percentage $2.87 \%$ and the in-control percentage $97.13 \%$, while the application is shown that in generalized variance chart, in the High process stage, test results indicate that the out-ofcontrol percentage $15.16 \%$ and the in-control percentage $84.84 \%$, while in the Low process stage, the out-of-control percentage $95.77 \%$ and in the Evaporation and Prilling stage, the out-of-control percentage $3.55 \%$ and the in-control percentage $96.45 \%$. These results allow us to determine whether the joint process variability is in-control or out-of-control. It is shown that the out-of-control and in-control percentage changes by using difference values of $r$. It was shown that there is a relationship between the value of $r$ and the out-of-control percentage, the out-of-control percentage increased by increasing the value of $r$. 


\section{Conclusion}

- The MCUSUM is shown that the MCUSUM chart is a multivariate

quality control chart, has the advantage of detecting small shifts in the process mean.

- The MCUSUM chart used to determine whether the process mean vector for two or more variables is in-control.

- The MCUSUM charts allow us to simultaneously monitor whether two or more related variables are in-control.

- Generalized variance chart used to determine whether or not the joint process variability (the joint variability that accounts for the variability of each charted variable) for two or variables is in-control.

- Generalized variance charts allow us to simultaneously monitor whether the joint variability of two or more related variables is incontrol.

Finally,

- The company should use MCUSUM quality control chart to monitor the quality of the urea production. Too, the company should use the MCUSUM chart to determine variables which causes the out-of-control signals.

- The company should use generalized variance chart to determine whether or not the joint process variability for two or more variables is in control.

\section{Acknowledgement}

The author is grateful to the associate editor and referees for careful reading and the Editor for very useful comments and suggestions. 


\section{References}

[1] B. M. Adams, C. A. Lowry and W. H. Woodall, The Use and Misuse of False Alarm Probabilities in Control Chart Design, In Frontiers in Statistical Quality Control 4, Edited by H. J. Lenz and G. B. Wetherill, The Wilrich, Physica-Verlag, Heidelberge, 1992.

[2] F. B. Alt, Multivariate Quality Control, Encyclopedia of the Statistical Sciences, John Wiley \& Sons, New York, 1985.

[3] F. B. Alt and N. D. Smith, Multivariate Process Control, Handbook of Statistics, P. R. Krishnaiah and C. R. Rao, Eds., North-Holland Elsevier Since Publishers B. 7 (1988), 333-351.

[4] L. C. Alwan, CUSM Quality Control Multivariate Approach, Communication inStatistics-Theory and Methods 15 (1986), 3531-3543.

[5] F. Anderson, An Introduction to Multivariate, Statistical Analysis, Wiley, 1958, 1984.

[6] F. Aparisi, J. Jabaloyes and A. Carrion, Statistical properties of the $|S|$ multivariate control chart, Commun. in Statist.-Theory and Methods 28(11) (1999), 2671-2686.

[7] G. A. Barnard, Control charts and stochastic processes, J. of the Royal Stat. Society (B) 21 (1959).

[8] D. Brook and D. A. Evan, An approach to the probability distribution of CUSUM run lengths, Biometrika 59 (1972), 539-549.

[9] R. B. Crosier, Multivariate generalizations of cumulative SUM quality control schemes, Technometrics 30 (1988), 291-303.

[10] R. B. Crosier, A new two-sided cumulative SUM quality control scheme, Technometrics 28 (1986), 187-194.

[11] M. A. Djauhari, Improved monitoring of multivariate process variability, J. of Quality Tech. 37(1) (2005), 32-39.

[12] E. Dogu and I. D. Kocakoc, Estimation of change point in generalized variance control chart, Commun. in Statist.: Simulation and Computation 40 (2011), 345-363.

[13] M. S. Hamed, Generalized variance chart for multivariate quality control process, Proc. with Appl. Appl. Math. Sci. 163(8) (2014), 8137-8151.

[14] D. M. Hawkins, Regression adjustment for variables in multivariate quality control, J. of Quality Tech. 25 (1993), 170-182.

[15] D. M. Hawkins, A fast approximation of average run lengths of CUSUM control charts, J. of Quality Tech. 24 (1992), 120-128.

[16] H. Hotelling, Multivariate Quality Control in Techniques of Statistical Analysis, McGraw Hill, New York, 1947, pp.111-184.

[17] A. Houshmand and A. Javaheri, Multivariate Ridge Residual Charts, Ph.D. Dissertation, University of Cincinnati, 1998. 
[18] J. E. Jackson, Multivariate quality control, Comm. in Stat.-Theo. and Methods 11 (1988), 2657-2688.

[19] J. E. Jackson and R. H. Morris, An application of multivariate quality control to photographic, Proc. J. of the Amer. Stat. Assoc. 52 (1957), 186-199.

[20] N. L. Johnson, A simple theoretical approach to cumulative SUM control charts, J. of the Amer. Stat. Assoc. 54 (1961).

[21] B. P. Korin, On the distribution of a statistic used for testing a covariance matrix, Biometrika 55(1) (1968), 171-178.

[22] W. Linna, H. Woodal and L. Busby, The performance of multivariate control charts in the presence of measurement error, J. of Quality Tech. 33 (2001), 133-139.

[23] D. C. Montgomery, Introduction to Statistical Quality Control, 4th Ed., John Wiley Sons, New York, NY, 2001.

[24] E. S. Page, Continuous inspection schemes, Biometrika 41 (1954), 100-114.

[25] J. J. Pignatiello and M. D. Kasunic, Development of multivariate CUSUM chart computers in engineering, The Amer. Society of Mech. Engin., New York (1985), 427-432.

[26] J. J. Pignatiello and G. C. Runger, Comparison of multivariate CUSUM charts, J. of Quality Tech. 22 (1990), 173-186.

[27] H. M. Shehata, Statistical Study of Multivariate Quality Control Procedures and its Applications, M.Sc. Thesis, Benha University, 2007.

[28] W. A. Shewhart, Economic Control of Quality of Manufactured Product, D. Van Nostrand Company INC, New York, 1931.

[29] D. Siegmund, Sequential Analysis: Tests and Confidence Intervals, Springer-Verlag, New York, 1985.

[30] N. D. Smith, Multivariate Cumulative Sum Control Charts, Ph.D. Dissertation, University of Matyland, College Park, 1987.

[31] L. C. Vance, Average run lengths of cumulative sum control charts for controlling normal means, J. of Quality Tech. 18 (1987).

[32] S. S. Wilks, Certain generalizations in the analysis of variance, Biometrika 24 (1932), 471-494.

[33] W. H. Woodall and B. M. Adams, The statistical design of CUSUM charts, Quality Engineering 5 (1993).

[34] W. H. Woodall and M. M. Ncube, Multivariate CUSUM quality-control procedures, Technometrics 27 (1985), 285-292. 